\title{
Presentación
}

\section{Medir la educación: perspectivas desde la crítica sociológica}

\author{
José Beltrán y António Teodoro'
}

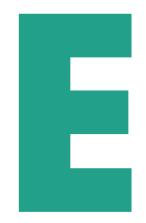

1 interés y la preocupación por una política educativa gobernada por los números se reflejan claramente en una literatura académica creciente desde disciplinas que en muchas ocasiones atraviesan fronteras epistemológicas y dialogan entre sí a nivel internacional. Si bien el problema acerca de la medida de la educación no es nuevo, es cierto que la generalización y proliferación de estudios, informes y pruebas que tratan de capturar resultados cuantitativos en la esfera educativa, estrechamente asociados a principios y criterios evaluativos, despierta tanto interés como inquietud. Recientemente se han desarrollado foros y debates que han planteado la necesidad de ofrecer alternativas a una corriente neopositivista que parece estar imponiéndose en las ciencias sociales, con efectos e impactos en las políticas y en las prácticas educativas. Así, en 2015, la revista Journal of Education Policy había prestado atención, en un número especial (vol. 30, n. ${ }^{\circ}$ 3), a «la educación, la gobernanza y la tiranía de los números», con un editorial firmado por Stephen J. Ball (2015:299-301), en el que advierte del papel singular que en la actualidad juegan las maneras de medir la educación dentro de la relación entre verdad y poder. Por su parte, la Sección de Educación Comparada de la Sociedad Portuguesa de Ciencias de la Educación (SPCE-SEC) celebró en Lisboa, en enero de 2016, una Conferencia Internacional en torno al tema «La Educación Comparada más allá de los números: contextos locales, realidades nacionales y procesos transnacionales», con el propósito de reflexionar sobre los modos de regulación de las políticas a través de grandes estudios internacionales. También a finales de 2016, Profesorado. Revista de curriculum y formación del profesorado editaba un monográfico (vol. 20, n. ${ }^{\circ}$ 3) con el título «políticas de evaluación y accountability en educación», coordinado por Almerindo Janela Afonso en cuya presentación reclamaba «una reflexión más densa» (2016:1-12). En enero de 2017 la revista Comparative Education, plantea un monográfico (vol. 53, n. ${ }^{\circ}$ 1) cuyo primer artículo, a cargo de Elaine Unterhalter (2017:1-16) cuestiona la pretensión de «medir lo inmensurable en educación». Estos ejemplos no pretenden agotar el inventario, pero sin duda constituyen un síntoma claro del abanico de problemas al que da paso la pregunta acerca de «la medida de la educación». No es casual, entonces, que la sociología de la educación, a través de la RASE, se muestre sensible a un debate que afecta, directa o indirectamente, a cuantos formamos parte de la comunidad educativa.

La discusión sobre los modos de medir la educación no es trivial. El debate se dirime en una tensión -si no en una clara oposición- entre la lógica y la dialéctica. Ambas son herramientas auxiliares legítimas

1 José Beltrán es profesor del Departament de Sociologia i Antropologia Social de la Universitat de València y miembro de RIAIPE. António Teodoro es Director del Centro de Estudos Interdisciplinares em Educação e Desenvolvimento (CeiED), de la Universidade Lusófona. Coordinador de la Red Iberoamericana de Investigación en Políticas Educativas (RIAIPE). 
y complementarias para comprender la realidad, pero no debería olvidarse que la validez científica no puede reducirse al valor numérico (ni tampoco al valor discursivo), y que hay aspectos de la realidad social que no son calculables: «¿cuánto vale una vida?» (García Ruíz, 2016:88), ¿cómo se mide una experiencia?, se preguntaba hace cien años John Dewey (1998 [1916]:287). No podemos contabilizar la intensidad de una discusión científica o de una argumentación racional, de la misma manera que no podemos discutir dialécticamente los resultados numéricos de una operación aritmética o una regla de la lógica formal. Tanto las teorías que elaboramos, como las hipótesis que sometemos a prueba y las creencias que tenemos están condicionadas por nuestra manera de medir, un término que está emparentado con la voz griega metron, y que significa trazar correctamente los propios límites, tener mesura (Grün, 2016:9). Por eso, desde el ámbito de la economía, de la política y de la sociología se están replanteando los sistemas de medición, en el sentido de cuestionar sus límites (Meyer, 2017:17-34), sus sesgos (Ozga, 2017), y sus posibilidades más allá del «espíritu de Arquímedes» (Sivesind, 2014:57-77).

Buena parte de las cuestiones que pivotan en torno a la medida de la educación están asociadas a la producción de indicadores, que está basada en la presunción de una suerte de superioridad epistemológica. En el caso de los indicadores de educación, está produciéndose un auténtico ascenso de «la métrica», favoreciendo la construcción de la figura de «expertos», que en ocasiones resultan ser «débiles» (Lim, 2017). Algunos de ellos prestan una atención cada vez mayor al impacto de la producción científica o académica en instituciones educativas, asociando de manera descontextualizada y desproporcionada calidad a cantidad. La proliferación en los últimos años de una serie de informes internacionales que se centran en la esfera educativa (PISA, PIRLS, TALIS...) refleja nuevos modos de regulación transnacional, sustentada en una evaluación de los resultados y de su comparación permanente a través de grandes encuestas e informes estadísticos. (Teodoro, 2010). Y esa industria contable contribuye a configurar a su vez, en el marco de la globalización hegemónica, un nuevo sentido común, una gobernanza epistémica. Todo ello nos devuelve, de nuevo, a la cuestión central, en la que se entrecruzan interés y conocimiento: ¿cómo se mide la educación?

Esta cuestión, a su vez, da paso a una serie de interrogantes: ¿Con qué propósitos (científicos, sociales, económicos...) medimos la educación? ¿Qué instrumentos se utilizan para ofrecer información sobre las diferentes variables del campo educativo? ¿Qué valor tiene la experiencia educativa? ¿Qué efectos tienen los informes que se emiten periódicamente desde las agendas educativas a nivel nacional e internacional? ¿Qué relación existe entre las mediciones educativas y las reformas educativas? ¿Qué marcos culturales e interpretativos subyacen a nuestros modos de medir la realidad educativa? ¿El papel de los sistemas de indicadores es informativo o también tiene consecuencias normativas? ¿Es necesaria o posible la construcción de indicadores alternativos a los que ahora mismo son dominantes?

Se trata de cuestiones que ya hemos planteado en anteriores trabajos cuando nos preguntábamos si los indicadores acaban poniendo más el foco en los productos que en los procesos (Beltrán y Villar, 2010). En una reflexión previa sobre evaluaciones internacionales (Teodoro y Montané, 2009), se advertía que los indicadores educativos no capturan hechos puros sino datos que ya se nos ofrecen filtrados por el propio artefacto de medición. Nada que objetar, siempre que no perdamos de vista esta diferencia. Desde el siglo pasado, como respuesta a las exigencias del empirismo lógico, se fundamentó la necesidad de contextualizar las representaciones de la realidad, evitando confundir el «mapa» con el «territorio». El autor que formuló esta distinción metafórica, Wittgenstein, comparó la aritmética con la institución bancaria, sosteniendo que podría desmoronarse en cuanto la gente desconfiara de ella y se apresurara a sacar de 
allí su dinero (Beltrán, 2009:11). No se pretende aquí cuestionar el valor de los números, sino más bien mostrar cautelas epistemológicas ante la suplantación de los significados por significantes, y de mensajes por los medios que les sirven de transporte. Ningún lenguaje es neutral en su uso ni es inocente en su aplicación. Desde un punto de vista social, no es posible ni deseable una descripción lógica aislada de una comprensión sociológica. La medida de la educación, al servicio de la lógica de la investigación científica, ha de explicar y explicarse en relación con prácticas sociales, con formas de vida. La superioridad epistemológica que se atribuye a la numérico (lo mensurable con números) se debe a su importante e innegable capacidad de abstracción, de separar elementos para su análisis, pero para la esfera de la educación no debemos olvidar la importancia de integrar estos elementos en un marco de análisis, crítico e interpretativo, más amplio, en una cultura común (superando de paso el abismo entre las dos culturas que ya denunciara en su momento C. P. Snow). Como toda tecnología al servicio de un fin determinado, la medida (de la educación) se puede considerar también un artefacto, un arma. Pero un arma tiene un significado ambivalente: puede convertirse en una herramienta constructiva, o puede convertirse en una amenaza, con efectos no previstos o no deseados. Todo depende del uso que decidamos darle. La dicotomía entre medición (lógica) y argumentación (dialéctica), puede superarse mediante una crítica sociológica que procure una «alianza tensa» con fines emancipadores. En este sentido, los artículos de esta revista ofrecen una buena muestra de los límites y posibilidades, de los usos y abusos, de las políticas y prácticas, así como de la creatividad e imaginación sociológica, a la hora de pensar y operativizar la medida de la educación.

Este monográfico reúne contribuciones que abordan el problema de la medida de la educación desde la sociología de la educación, con el concurso de otras disciplinas cercanas y convergentes, a través de una serie de miradas críticas y de enfoques plurales. Sin duda, las contribuciones que aquí se dan cita pueden estimular y enriquecer la discusión científica y el debate académico en torno a las implicaciones sociales de nuestros modos de medir el campo educativo en sus múltiples facetas.

En este número participan, con trece artículos y dos reseñas, veintiséis investigadoras e investigadores procedentes de nueve países de diferentes regiones del mundo: Brasil, México, Uruguay, Estados Unidos, Australia, Rusia, Alemania, Portugal y España. Las aportaciones para este número monográfico de la RASE exploran un conjunto de dimensiones muy variadas en torno al tema propuesto de la medida de la educación. Algunas adoptan una perspectiva principalmente teórica, abordando cuestiones acerca de la validez científica, la fundamentación epistemológica y las implicaciones sociales y políticas en la concepción y en el modo de medir la educación; otras tienen un carácter más empírico, y presentan resultados a partir de investigaciones ya realizadas o en curso que se han servido de herramientas de medición educativa; y también, en buena parte de los textos se combinan las perspectivas teóricas y empíricas al servicio de análisis que ilustran diferentes metodologías, aplicaciones y efectos, en ningún caso neutrales, de la medida de la educación. Estos tres tipos de enfoques diferenciados, que sirven a modo de clasificación, es el que nos ha facilitado el criterio de presentación y ordenación de los artículos.

En un primer bloque, de orientación principalmente teórica, se reúnen cuatro artículos. En el primero de ellos, Nelly P. Stromquist, partiendo de la realidad estadounidense pero más allá de la misma, lleva a cabo una crítica fundamentada sobre los efectos no previstos de la aplicación de los test estandarizados y cuestiona la supuesta relación entre la calidad educativa y su medición. Por su parte, Alexander Osipov propone un giro cualitativo, argumentando en su análisis la necesidad de un enfoque sobre las funciones de la educación considerada como institución social, en tanto que nexo teórico para dimensionarla y operativizarla conceptualmente. Dietmar Pfeiffer, en su texto, subraya el carácter ambiguo de los indicadores 
más extendidos, la descontextualización en su aplicación, al tiempo que analiza la confusión común entre medir (un acto descriptivo) y evaluar (un acto normativo) y observa la configuración de una «cultura de los indicadores» que actúa como mecanismo de control. Desde un punto de vista poscolonial, Almerindo Janela Afonso cuestiona y denuncia la hegemonía epistémica eurocéntrica que reflejan los programas de evaluación comparativa internacional, subordinados a una agenda global asimétrica en sus intereses y en sus relaciones de poder.

En un segundo apartado, se dan cita una serie de cinco textos en los que prevalece la orientación empírica. Así, Rubia Fonseca, Joaquim Escola, Amâncio Carvalho y Armando Loureiro presentan un estudio comparativo en el que se analizan, sirviéndose de diferentes escalas de medición, las competencias de estudio de los estudiantes universitarios de universidades de Brasil y Portugal, con resultados sobre sus condiciones, actitudes y estrategias. El trabajo en equipo de Sandra Saúde, Sandra Lopes, Carlos Borralho e Isidro Féria, contextualizado en Portugal y en el caso del sistema politécnico de educación superior, se centra en el valor y las tensiones que pueden suponer la introducción de nuevas formas de medir el desempeño profesional en la dinámica educativa y organizativa, a partir de un proyecto en el que se ensayan la aplicación de una serie de indicadores. Ian Hardy reflexiona sobre cómo la formación continua de maestras y maestros está condicionada por una creciente medición de los resultados de aprendizaje de los estudiantes. Su estudio se centra en una escuela de Australia y en el análisis de una serie de reuniones en las que los docentes expresan cómo la manera de medir el aprendizaje afecta y altera su propia formación docente. A continuación, Uyguaciara Veloso Castelo Branco, Paulo Hideo Nakamura y Edineide Jezine presentan la elaboración de una escala de permanencia discente, sirviéndose de la Lógica Paraconsistente, en el marco de un proyecto sobre la permanencia en la Educación Superior en Brasil. Desplazando el punto de enfoque, Armando Alcántara presta atención al modo de medir la investigación a través del estudio de las revistas de educación iberoamericanas. El artículo presenta los primeros resultados de un proyecto que analiza los efectos de la información bibliométrica en las revistas académicas.

Finalmente, cuatro nuevas entregas completan el tercer bloque de esta revista, con artículos elaborados desde aproximaciones teórico-empíricas. Daniel A. Wagner y Nathan M. Castillo ofrecen un análisis de las tensiones científicas en la comprensión del aprendizaje en poblaciones pobres y marginadas, que se sitúan en la base de la pirámide. Los autores argumentan la necesidad de que las Naciones Unidas prioricen el aprendizaje de la población empobrecida con el fin de que los objetivos para el desarrollo post-2015 aborden de manera crítica y comprometida las inequidades sociales y económicas. Desde una óptica distinta, Regina Célia Linhares Hostins aborda las políticas educativas de evaluación de postgrado implementadas en la reforma educativa neoliberal de Brasil en los años noventa. Y lo hace en una investigación de análisis documental inspirada en la teoría de la interpretación-traducción de la política propuesta, entre otros, por Stephen Ball. Cambiando de nuevo la mirada, Carmen Carmona Rodríguez se sirve del documental Tested (2015) -centrado en la aplicación en la ciudad de Nueva York del polémico Specialized High School Admission Test (SHSAT) dirigido a estudiantes de entre 12-13 años- para reflexionar sobre las consecuencias en términos de distribución de oportunidades que tiene la realización de evaluaciones estandarizadas al alumnado. Por último, Alejandra Montané, José Beltrán y António Teodoro revisan la construcción de sistemas transnacionales de evaluación de la calidad, a través de los principales rankings internacionales, desde una perspectiva crítica. Los autores sostienen que bajo el modelo de la «calidad educativa» se ha legitimado el dominio de una poderosa industria contable, alejada del horizonte de equidad y de justicia social, cognitiva y curricular. 
El panorama de perspectivas que ofrecen todas estas contribuciones desde la sociología de la educación supone una muestra de la necesidad de llevar a cabo reflexiones que se puedan nutrir al mismo tiempo de la lógica de la investigación científica y de la dialéctica social. Inspirándose en ambas, la crítica sociológica invita, además, a dar el paso desde el compromiso académico a la acción social fundamentada.

La escritura compartida en el espacio que brinda una revista también es una forma de conversación y de acción. Los editores quieren agradecer a todos y cada uno de quienes han hecho posible este número, con sus valiosos trabajos, de manera desinteresada, y más allá de sus obligaciones profesionales.

\section{Referencias bibliográficas}

Afonso, Almerindo Janela (2016): "El campo de las políticas de accountability en educación. Para una reflexión más densa". Profesorado. Revista de curriculum y formación del profesorado, 20 (3), 1-12.

Ball, Stephen J. (2015): "Education, governance and the tyranny of numbers". Journal of Education Policy, 30 (3), 299-301.

Beltrán, José (2009): "Presentación” en António Teodoro y Alejandra Montané (coord.): Espejo y reflejo: Políticas curriculares y evaluaciones internacionales. Alzira, Germania.

Beltrán Llavador, José y Villar Aguilés, Alícia (2010): “La medida de la educación: algunas consideraciones a propósito de los indicadores”. Educação \& Linguagem, 13, (21), 134-149.

Dewey, John ([1916]1998). Democracia y educación. Madrid, Morata.

García Ruíz, Alicia (2016). Impedir que el mundo se deshaga. Para una emancipación ilustrada. Barcelona, Catarata.

Grün, Anselm (2016). El arte de la justa medida. Madrid, Trotta.

Lim, Miguel Antonio (2017): “The building of weak expertise: the work of global university rankers". High Education. $13^{\text {th }}$ of April.

Meyer, Heinz-Dieter (2017): “The limits of measurement: misplaced precision, phronesis, and other Aristotelian cautions for the makers of PISA, APPR, etc.”. Comparative Education, 53 (1), 17-34.

Ozga, Jenny (2017): "Education policy should not be driven by performance data". Nature Human Behaviour, $1,0014$.

Sivesind, Kirsten (2014): "Education in the Spirit of Archimedes: pertaining to the buoyancy of PISA" en Andreas Nordin \& Daniel Sundberg (ed.): Transnational Policy Flows in European Education: the making of knowledge in the education policy field. Oxford: Symposium Books.

Teodoro, António y Montané, Alejandra (coord.) (2009). Espejo y reflejo: Políticas curriculares y evaluaciones internacionales. Alzira: Germania.

Teodoro, António (2010). Educação, Globalização e Neoliberalismo. Os novos modos de regulaşão transnacional das políticas de educação. Lisboa: Edições Universitarias Lusófonas.

Unterhalter, Elaine (2017): "Negative capability? Measuring the unmeasurable in education". Comparative Education, $53(1), 1-16$. 